\title{
Molecular Modeling and Spectroscopic Studies on the Interaction of Transresveratrol with Bovine Serum Albumin
}

\author{
Xiaoli Liu, ${ }^{1,2}$ Yonghui Shang, ${ }^{3}$ Xudong Ren, ${ }^{4}$ and Hua $\mathrm{Li}^{4}$ \\ ${ }^{1}$ College of Life Sciences, Northwest University, Xian 710069, China \\ ${ }^{2}$ College of Environmental Science and Engineering, Chang'an University, Xian 710054, China \\ ${ }^{3}$ Department of Chemistry, Xianyang Normal College, Xianyang 712000, China \\ ${ }^{4}$ College of Chemistry and Materials Science, Northwest University, Xian 710069, China \\ Correspondence should be addressed to Hua Li; nwufxkx2012@126.com
}

Received 7 January 2013; Revised 25 February 2013; Accepted 2 March 2013

Academic Editor: André Silva Pimentel

Copyright (c) 2013 Xiaoli Liu et al. This is an open access article distributed under the Creative Commons Attribution License, which permits unrestricted use, distribution, and reproduction in any medium, provided the original work is properly cited.

\begin{abstract}
The interaction of transresveratrol (TRES) with bovine serum albumin (BSA) has been investigated by ultraviolet-visible, fluorescence, Fourier transform infrared spectroscopic methods and molecular modeling techniques. The fluorescence results show that the intrinsic fluorescence of BSA is quenched by TRES through a static quenching procedure. The binding constants of TRES with BSA at 292,297 and $302 \mathrm{~K}$ are calculated as $10.22 \times 10^{4}, 8.71 \times 10^{4}$, and $7.59 \times 10^{4} \mathrm{~L} \mathrm{~mol}^{-1}$, respectively, and corresponding numbers of binding sites are approximately equal to unity. The thermodynamic parameters $\Delta H$ and $\Delta S$ are estimated to be $-21.82 \mathrm{~kJ} \mathrm{~mol}^{-1}$ and $+21.15 \mathrm{~J} \mathrm{~mol}^{-1} \mathrm{~K}^{-1}$, which indicates that the interaction of TRES with BSA is driven mainly by hydrophobic forces and there are also hydrogen bonds and electrostatic interactions. The competitive experiments suggest that the binding site of TRES to BSA is probably located on site II. The results of infrared spectra show that the binding of TRES with BSA leads to conformational changes of BSA, and the binding stabilizes the $\alpha$-helix and $\beta$-sheet at the cost of a corresponding loss in the $\beta$-turn structure of BSA. The results of molecular modeling calculation clarify the binding mode and the binding sites which are in good accordance with the experiment results.
\end{abstract}

\section{Introduction}

Serum albumins, the major soluble protein constituents in the blood stream, bind and transport several exogenous and endogenous molecules like fatty acids, nutrients, steroids, and a large list of drugs. The formed complexes are involved in transport and regulatory processes. Since most of the administered drugs bind extensively and reversibly to serum albumins and drugs are transported mainly as complexes with proteins, the biological activities of the drug such as the overall distribution, metabolism, and efficacy in the body are correlated with their affinities towards serum albumins. Therefore, protein-drug interaction has an enormous biological interest and has been found to play a significant role in pharmacology [1]. Among the serum albumins, bovine serum albumin (BSA) is an appropriate protein model for studying the interaction between serum albumins and drugs because of its medically important, unusual ligand-binding properties, low cost, availability, and structural homology with human serum albumin (HSA) [2,3].

Transresveratrol (trans-3,5, $4^{\prime}$-trihydroxystilbene, TRES) is a polyphenolic natural product existing in a variety of plant species including Polygonum cuspidatum, grapes, peanuts, and various berries. TRES has been shown to prevent or slow the progression of a wide variety of diseases, including cancer, cardiovascular disease, ischemic injuries, and Alzheimer's disease [4-8]. These properties accompanied with the lack of harmful effects make TRES attractive as a phytoalexin. The molecular structure of TRES is provided in Figure 1. So far, most researches are focusing on its pharmacological activities; only a little has been done on the interaction of TRES with serum albumin. Li et al. [9], Wei et al. [10], and Cao et al. [11] had reported the interaction between resveratrol and BSA, but the binding site of TRES on BSA had not been identified, the changes of secondary structure of BSA had not been quantitatively analyzed, and the binding 


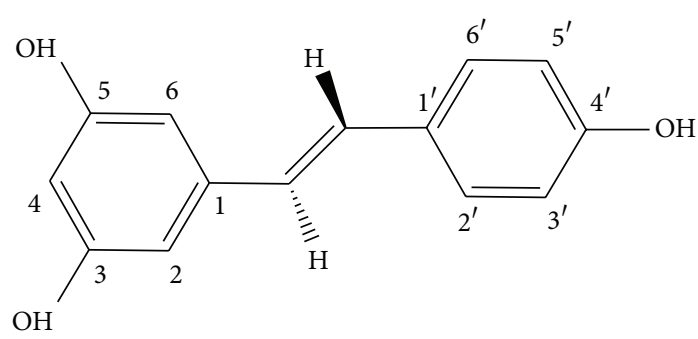

FIgURE 1: Molecular structure of TRES.

had not been calculated by molecular modeling techniques. In this paper, the interaction of TRES with BSA is investigated in detail with the purpose to provide basic information for pharmaceutical applications of TRES.

In this work, the interaction of TRES with BSA has been investigated under imitated physiological conditions via spectroscopic methods and molecular modeling techniques. Fluorescence and UV-vis spectroscopy were employed to understand the quenching mechanism of BSA-TRES binding. The binding constants, number of binding sites, and thermodynamic parameters have been calculated, and the binding site of TRES on BSA was identified by fluorescence displacement experiments. Furthermore, Fourier transform infrared (FTIR) spectroscopy was used to demonstrate the conformational changes of BSA, and the changes of secondary structure were quantitatively analyzed by the curve fitting method. In addition, the molecular modeling was used to improve the understanding of the interaction of TRES with BSA.

\section{Experimental}

2.1. Materials. BSA was purchased from Huamei Bioengineering Company (Shanghai, China). TRES was obtained from Xi'an Sino-Herb Biotechnology Company (Xian, China). All other reagents were analytical-reagent grade, and double-distilled water was employed for solutions.

2.2. Fluorescence and UV-Vis Experiments. The fluorescence experiments were performed with an F-4500 fluorescence spectrophotometer (Hitachi). For different samples, the concentrations of BSA were constant at $3 \times 10^{-7} \mathrm{~mol} \mathrm{~L}^{-1}$ and the concentrations of TRES were alterable and ranged from 0 to $2.4 \times 10^{-6} \mathrm{~mol} \mathrm{~L}^{-1}$. Fluorescence quenching spectra were measured at 292, 297 and $302 \mathrm{~K}$ with exciting wavelength of $280 \mathrm{~nm}$. The UV-vis absorption experiments were performed on a UV-2450 spectrophotometer (Shimadzu, Japan).

2.3. Displacement Experiments. The displacement experiments were performed using three different site probes, namely, phenylbutazone, flufenamic acid, and digitoxin, for site I, II, and III, respectively. BSA and the site probe were added to colorimeter tube first, and the concentrations were constant at $3 \times 10^{-7} \mathrm{~mol} \mathrm{~L}^{-1}$ and $2 \times 10^{-7} \mathrm{~mol} \mathrm{~L}^{-1}$, respectively. Then appropriate amount TRES was added to the BSAphenylbutazone/flufenamic acid/digitoxin systems.
2.4. FTIR Experiments. FTIR spectra were measured on a Vertex 70 FTIR spectrometer (Bruker Optics, Germany). All FTIR spectra were taken via the attenuated total reflection method. The spectra processing procedure involved collecting spectra of Tris- $\mathrm{HCl}$ buffer solution, BSA solution, TRES solution, and BSA-TRES solution under the same conditions. Next, the absorbance of the buffer solution was subtracted from the spectrum of the BSA solution to obtain the spectrum of free BSA, and the absorbance of the TRES solution was subtracted from the spectrum of the BSA-TRES solution to obtain the spectrum of the BSA after interaction. At last, the two difference spectra were compared and analyzed.

2.5. Molecular Modeling. The crystal structure of BSA was obtained from protein databank (PDB) (http://www.rcsb.org/pdb, PDB ID: 3V03), the PDB file was imported into AutoDock Tools, all water molecules were removed, and Kollman charges and solvation parameters were added. The initial 3D structures of TRES were generated by ChemSkcteh software, and the geometries of TRES were subsequently optimized using GAMESS software on DFT/B3LYP/6-31G level. AutoDock4.2 (http://autodock.scripps.edu) program was applied to calculate the interaction modes between TRES and BSA. First the grid box was set as $126 \times 100 \times 126$ points with a grid spacing of $0.560 \AA$, whole protein was covered, and the initial search was carried out. The region of the most populated of the first five clusters was selected as the probable binding region. Then the accurate search was executed in the probable binding region with a smaller grid map. Lamarckian genetic algorithm was applied in the docking. 100 genetic algorithm (GA) runs were performed with the following parameters: population size of 150, maximum number of $2.5 \times 10^{6}$ energy evaluations, and maximum number of 27,000 generations, and other parameters were default. The resulting conformations were clustered using a root-meansquare deviation (RMSD) of $2.0 \AA$ and the clusters were ranked in order of increasing binding energy of the lowest binding energy conformation in each cluster. Finally the lowest energy conformation in the most populated of the first five clusters was used for docking analysis. The output from AutoDock was rendered with PyMOL.

\section{Results and Discussion}

3.1. Fluorescence Quenching Mechanism. The fluorescence spectra of BSA in the presence of different concentrations of TRES are shown in Figure 2. It is obvious that BSA has a strong fluorescence emission peak at about $347 \mathrm{~nm}$, and the fluorescence intensity decreases gradually with increasing concentration of TRES, which indicates that TRES can interact with BSA and quench its intrinsic fluorescence. Furthermore, there is a slight red shift of maximum emission wavelength occurring with the addition of TRES, implying that the microenvironment around the chromophore of BSA is changed.

Fluorescence quenching can occur by different mechanisms, usually classified as dynamic quenching and static 
TABLE 1: Stern-Volmer quenching constants for the interaction of TRES with BSA at different temperatures.

\begin{tabular}{cccc}
\hline$T / K$ & $K_{\text {sv }}\left(\mathrm{L} \mathrm{mol}^{-1}\right)$ & $K_{q}\left(\mathrm{~L} \mathrm{~mol}^{-1} \mathrm{~s}^{-1}\right)$ & Correlation coefficient \\
\hline 292 & $7.36 \times 10^{4}$ & $7.36 \times 10^{12}$ & 0.9978 \\
297 & $5.27 \times 10^{4}$ & $5.27 \times 10^{12}$ & 0.9921 \\
302 & $4.64 \times 10^{4}$ & $4.64 \times 10^{12}$ & 0.9960 \\
\hline
\end{tabular}

quenching. In order to clarify the quenching mechanism of TRES on BSA, the Stern-Volmer equation (1) is used to analyze the fluorescence quenching data [12], where $F_{0}$ and $F$ are the fluorescence intensities in the absence and presence of quencher, $K_{q}$ is the quenching rate constant of the biomolecule, $\tau_{0}$ is the average lifetime of the biomolecule in the absence of quencher which equals $10^{-8} \mathrm{~s}$ [13], $K_{\mathrm{SV}}$ is the Stern-Volmer quenching constant, and $[Q]$ is the concentration of quencher. One has

$$
\frac{F_{0}}{F}=1+K_{q} \tau_{0}[Q]=1+K_{\mathrm{sv}}[Q] .
$$

Within certain concentration, the curve of $F_{0} / F$ versus [Q] would be linear if the quenching type is single static or dynamic process. The Stern-Volmer curves of TRES with BSA at three different temperatures exhibit good linear relationship within the investigated concentrations, so the values of $K_{q}$ and $K_{\mathrm{SV}}$ can be determined from the slope of regression curve of $F_{0} / F$ versus $[Q]$, and the results are listed in Table 1. For dynamic quenching, the maximum scatter collision quenching constant of various quenchers with the biopolymers is $2.0 \times 10^{10} \mathrm{~L} \mathrm{~mol}^{-1} \mathrm{~s}^{-1}$. In addition, dynamic and static quenching can be distinguished by temperature dependence of the quenching, the values of $K_{\mathrm{SV}}$ decrease with an increasing temperature for static quenching and the reverse result for dynamic quenching. From Table 1, the results show that the values of $K_{\mathrm{SV}}$ and $K_{q}$ decrease with the increasing of temperature and the values of $K_{q}$ is much greater than $2.0 \times 10^{10} \mathrm{~L} \mathrm{~mol}^{-1} \mathrm{~s}^{-1}$, which indicates that the probable quenching mechanism of BSA-TRES interaction is initiated by static quenching rather than by dynamic collision $[14,15]$. The results are also confirmed by UV-vis experiments as shown in Figure 3. Figure 3 illustrates that the UV-vis absorption spectrum of BSA and the difference absorption spectrum between BSA-TRES and TRES at the same concentration could not be superposed. After interaction with TRES, the absorbance band intensity of BSA at $280 \mathrm{~nm}$ decreases and the peak position shows a slight blue shift, suggesting the formation of BSA-TRES complex.

3.2. The Binding Constant and Number of Binding Sites. When small molecules bind independently to a set of equivalent sites on a macromolecule, the binding constant $\left(K_{b}\right)$ and the number of binding sites $(n)$ can be obtained from fluorescence intensity data by [16]

$$
\log \left[\frac{\left(F_{0}-F\right)}{F}\right]=\log K_{b}+n \log [Q] .
$$

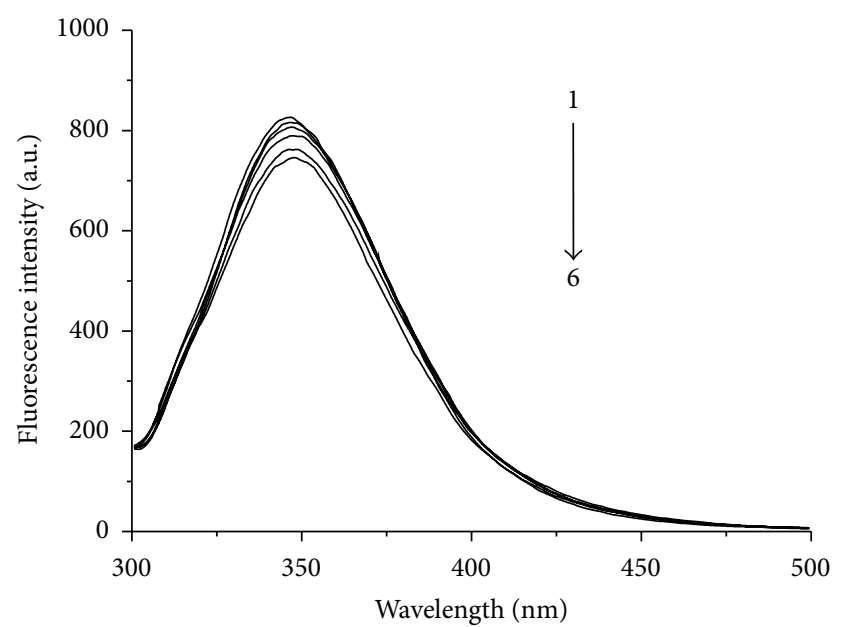

FIGURE 2: Fluorescence quenching spectra of BSA in presence of different concentrations of TRES. $C_{\mathrm{BSA}}=3.0 \times 10^{-7} \mathrm{~mol} \mathrm{~L}^{-1} ; C_{\mathrm{TRES}}$ $\left(\times 10^{-7} \mathrm{~mol} \mathrm{~L}^{-1}\right)(1-6): 0,3,6,12,18$, and 24 , respectively; $\lambda_{\mathrm{ex}}=$ $280 \mathrm{~nm} ; T=302 \mathrm{~K}$.

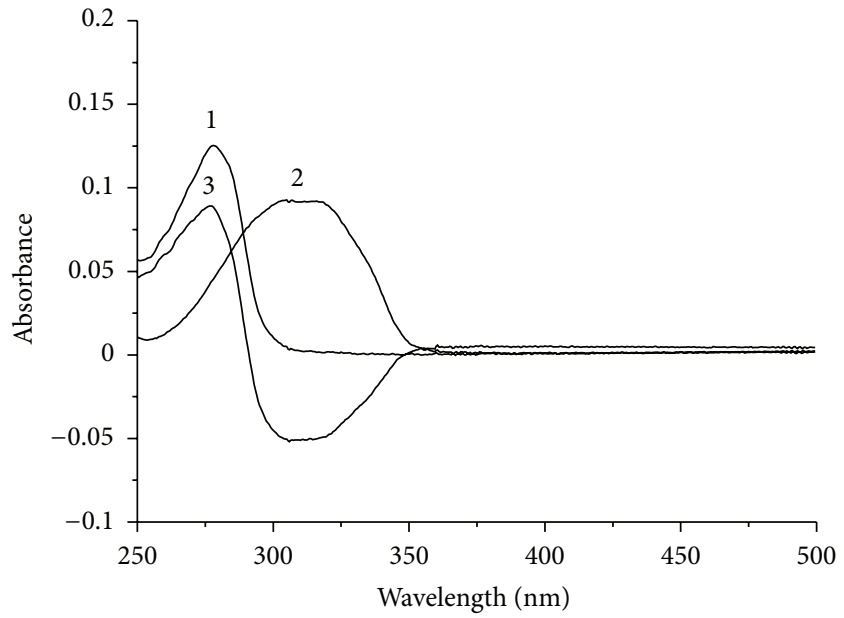

FIGURE 3: UV-vis absorption spectra of BSA (1), TRES (2), and the difference spectrum between TRES-BSA and TRES (3). $C_{\mathrm{BSA}}=$ $C_{\text {TRES }}=3.0 \times 10^{-5} \mathrm{~mol} \mathrm{~L}^{-1} ; \mathrm{pH}=7.4$.

Based on (2), the values of $n$ and $K_{b}$ can be determined from the slope and intercept of the double logarithm regression curve $\left(\log \left[\left(F_{0}-F\right) / F\right]\right.$ versus $\left.\log [Q]\right)$, and the corresponding calculated results are listed in Table 2 . It is found that the values of $K_{b}$ decrease with the increasing of temperature, indicating that the stability of BSA-TRES complex reduces with the temperature rising. The values of $n$ approximately equal unity, indicating that there is one independent class of binding sites in BSA for TRES.

3.3. Thermodynamic Analysis and Binding Mode. Thermodynamic parameters relying on temperatures are analyzed to characterize the acting forces between TRES and BSA. Generally speaking, the binding forces between drugs and biomolecules mainly include electrostatic interaction, hydrogen bond, van der Waals interaction, and hydrophobic 


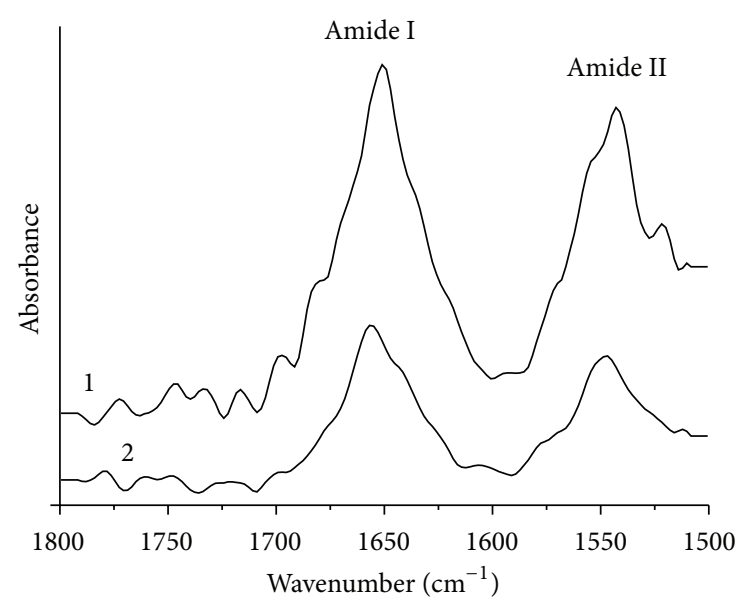

FIgURE 4: FTIR difference spectra of BSA in Tris- $\mathrm{HCl}$ buffer solution. (1) free BSA $\left(C_{\mathrm{BSA}}=1.0 \times 10^{-4} \mathrm{~mol} \mathrm{~L}^{-1}\right)$; (2) bonded BSA $\left(C_{\mathrm{BSA}}=1.0 \times 10^{-4} \mathrm{~mol} \mathrm{~L}^{-1}, C_{\mathrm{TRES}}=2.0 \times 10^{-4} \mathrm{~mol} \mathrm{~L}^{-1}\right) \cdot \mathrm{pH}=7.4$.

interaction [17]. Thermodynamic parameters, free energy change $(\Delta G)$, enthalpy change $(\Delta H)$, and entropy change $(\Delta S)$ of interaction, are essential to interpret the binding mode of BSA-drugs complexes. When enthalpy change $(\Delta H)$ does not vary significantly in the temperature range studied, both enthalpy change $(\Delta H)$ and entropy change $(\Delta S)$ can be determined from van't Hoff equation (3). In (3), $K$ corresponds to the binding constant at specific temperature and $R$ is the gas constant. $\Delta H$ and $\Delta S$ can be calculated from the slope and intercept of the regression curve of $\ln K$ versus $1 / T . \Delta G$ is consequently obtained according to (4). One has

$$
\begin{aligned}
\ln K & =\frac{-\Delta H}{R T}+\frac{\Delta S}{R}, \\
\Delta G & =\Delta H-T \Delta S .
\end{aligned}
$$

The Values of $\Delta H, \Delta S$, and $\Delta G$ for the interaction of TRES with BSA are presented in Table 2. The negative value of $\Delta G$ indicates the binding process is spontaneous. Positive value of $\Delta S$ is evidence of hydrophobic interactions [18]. In addition, specific electrostatic interaction between ionic species in aqueous solution is also characterized by a positive $\Delta S$ and a negative $\Delta H$. Generally, $\Delta G$ mainly comes from the large contribution of $\Delta S$ with little contribution from $\Delta H$ for electrostatic interaction [19]. However, in this work, $\Delta G$ mainly comes from the contribution of $\Delta H$. Therefore, we infer that hydrophobic interaction might play a major role in the interaction of TRES with BSA, and electrostatic force may also be involved in the binding process. Meanwhile, hydrogen bond can not be excluded as TRES is a polyphenolic compound.

3.4. Identification of Binding Site. Similar to HSA, the globular protein BSA consists of three distinct drug-binding sites [20]. Sudlow et al. have suggested that site I of serum albumin shows affinity for warfarin, phenylbutazone, and so forth and site II for ibuprofen, flufenamic acid, and so forth [21]. Later studies indicated that digitoxin binding is independent
TABLE 2: Binding constants and thermodynamic parameters of BSATRES interaction at different temperatures.

\begin{tabular}{cccccc}
\hline$T / K$ & $\begin{array}{c}K_{b} \\
\left(\mathrm{~L} \mathrm{~mol}^{-1}\right)\end{array}$ & $n$ & $\begin{array}{c}\Delta H \\
\left(\mathrm{~kJ} \mathrm{~mol}^{-1}\right)\end{array}$ & $\begin{array}{c}\Delta S \\
\left(\mathrm{~J} \mathrm{~mol}^{-1} \mathrm{~K}^{-1}\right)\end{array}$ & $\begin{array}{c}\Delta G \\
\left(\mathrm{~kJ} \mathrm{~mol}^{-1}\right)\end{array}$ \\
\hline 292 & $10.22 \times 10^{4}$ & 1.03 & & & -28.00 \\
297 & $8.71 \times 10^{4}$ & 1.03 & -21.82 & 21.15 & -28.10 \\
302 & $7.59 \times 10^{4}$ & 1.04 & & & -28.21 \\
\hline
\end{tabular}

TABLE 3: Binding constants of competitive experiments $(T=302 \mathrm{~K}$, $\lambda_{\text {ex }}=280 \mathrm{~nm}$ ).

\begin{tabular}{lccc}
\hline Site marker & $K_{b}\left(\mathrm{~L} \mathrm{~mol}^{-1}\right)$ & $R$ & $n$ \\
\hline Blank & $7.59 \times 10^{4}$ & 0.9973 & 1.03 \\
phenylbutazone & $6.17 \times 10^{4}$ & 0.9860 & 1.01 \\
flufenamic acid & $1.17 \times 10^{4}$ & 0.9794 & 0.85 \\
digitoxin & $6.11 \times 10^{4}$ & 0.9899 & 0.98 \\
\hline
\end{tabular}

of sites I and II and binds to site III [22]. To identify the binding site of TRES on BSA, competition experiments were carried out with phenylbutazone, flufenamic acid, and digitoxin as site probes for site I, II, and III, respectively [23, 24]. According to (2), the experiment data are analyzed and the binding constants of BSA-TRES system in the presence of different site markers are calculated in Table 3. Obviously, flufenamic acid could significantly influence the binding of TRES with BSA, whereas phenylbutazone or digitoxin has a lesser effect on the binding of TRES to BSA. Hence, it can be concluded that TRES is mainly bound to site II (subdomain IIIA) of BSA.

3.5. Conformation Investigation. To verify whether the conformation of BSA had changed or not in the absence and presence of TRES, FTIR measurements were performed. FTIR spectra of proteins exhibit a number of amide bands due to different vibrations of the peptide moiety. The amide I peak position occurs in the region $1600-1700 \mathrm{~cm}^{-1}$ (mainly $\mathrm{C}=\mathrm{O}$ stretch) and amide II band in the region $1500-1600 \mathrm{~cm}^{-1}$ (C-N stretch coupled with $\mathrm{N}-\mathrm{H}$ bending mode). They both have a relationship with the secondary structure of proteins, and amide I band is more sensitive to the change of proteins secondary structure than amide II [25]. The FTIR difference spectra of free BSA and bonded BSA (after binding with TRES) are shown in Figure 4. It is noticed that the peak position of amide I moves from 1651 to $1655 \mathrm{~cm}^{-1}$, and that of amide II shifts from 1541 to $1545 \mathrm{~cm}^{-1}$ in the FTIR spectrum of BSA after addition of TRES. From the shift in peaks position we can confirm that the conformation of BSA has been affected by the addition of TRES.

According to the literature [26,27], The spectral ranges $1610-1640 \mathrm{~cm}^{-1}, 1640-1650 \mathrm{~cm}^{-1}, 1650-1659 \mathrm{~cm}^{-1}$, and $1660-$ $1700 \mathrm{~cm}^{-1}$ in the amide I are assigned generally to $\beta$-sheet, random coil, $\alpha$-helical, and $\beta$-turn structure, respectively. To analyze quantitatively the protein secondary structure, the infrared self-deconvolution and second derivative resolution enhancement were applied to estimate the number, position, 


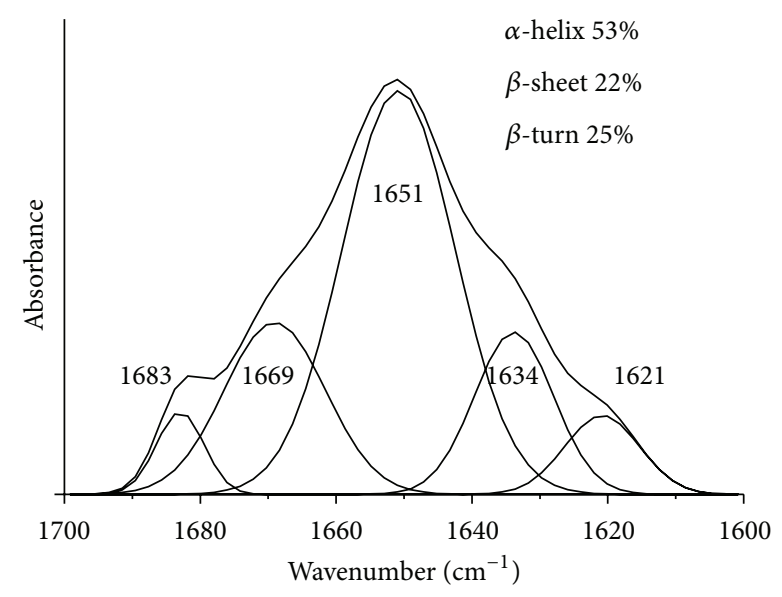

(a)

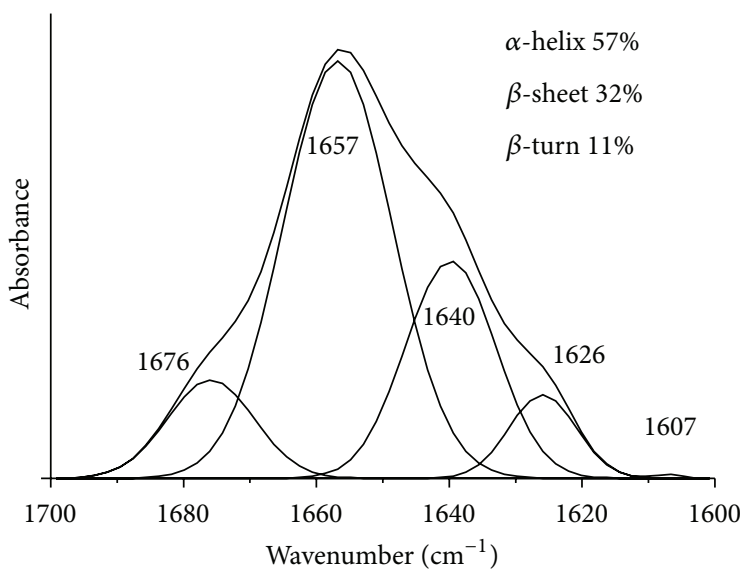

(b)

Figure 5: Curve-fitted amide I region for FTIR difference spectra of BSA. (a) Free BSA $\left(C_{\mathrm{BSA}}=1.0 \times 10^{-4} \mathrm{~mol} \mathrm{~L}^{-1}\right)$; (b) bonded BSA $\left(C_{\mathrm{BSA}}=\right.$ $\left.1.0 \times 10^{-4} \mathrm{~mol} \mathrm{~L}^{-1}, C_{\text {TRES }}=2.0 \times 10^{-4} \mathrm{~mol} \mathrm{~L}^{-1}\right) \cdot \mathrm{pH}=7.4$.

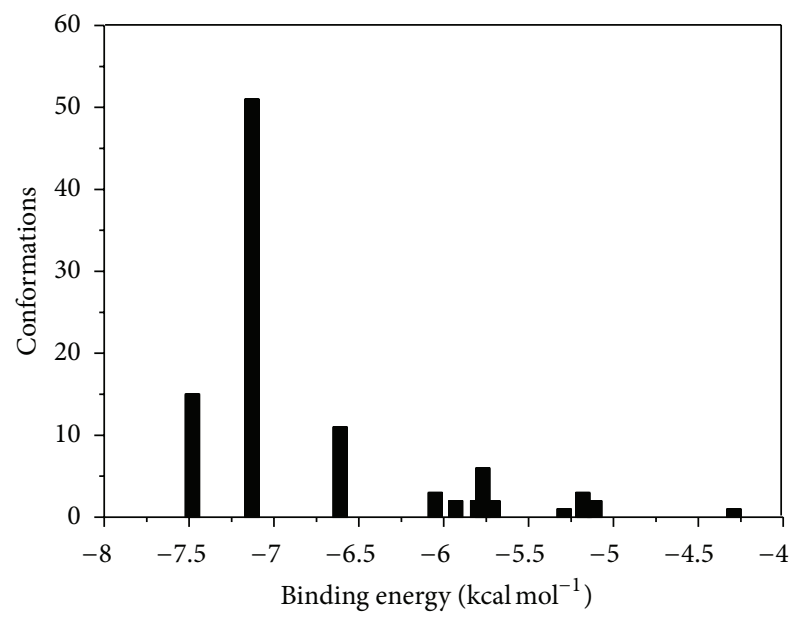

FIGURE 6: The clustering histogram at site II (RMSD: $2.0 \AA$ ).

and width of component bands in the region of 1700$1600 \mathrm{~cm}$; then curve fitting procedures were used to get the best Gaussian-shaped curves that fit the original protein spectrum. The percentage of each secondary structure of BSA can be calculated based on the integrated areas of the component bands in amide I, and the results are shown in Figure 5. The results show that before and after the interaction with TRES, the protein major secondary structures had changed, $\alpha$-helical structure increased from $53 \%$ to $57 \%, \beta$ sheet increased from $22 \%$ to $32 \%$, and $\beta$-turn structures decreased from $25 \%$ to $11 \%$, respectively.

3.6. Molecular Modeling Study. The fluorescence, UV-vis, and FTIR spectroscopic results were complemented with molecular modeling in which TRES was docked to BSA to determine the preferred binding site and the binding mode $[28,29]$. In the initial exploration, the results predict that compared with site I and III, the TRES was mostly concentrated in site II with lower binding energy, so site II was selected as the probable

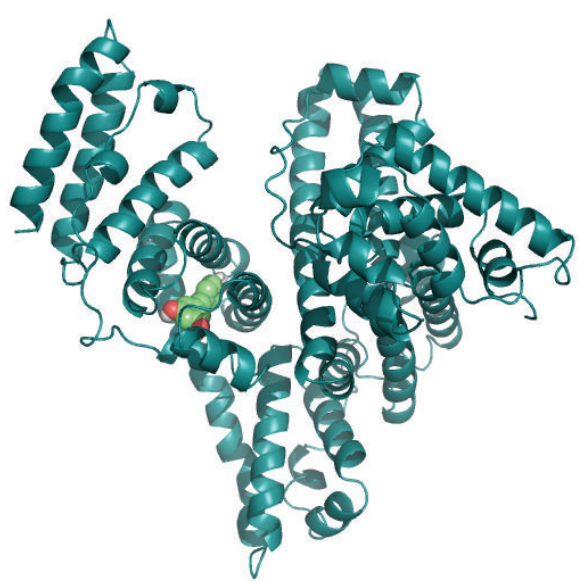

FIGURE 7: Best conformation for TRES docked to BSA. BSA is represented using cartoon ribbons and TRES is represented using ball.

binding region. Then the further exploration was carried out with a smaller grid map of $86 \times 82 \times 72$ points centered at $39.148,20.061$, and 53.329 with a spacing of $0.375 \AA$ at site II. The clustering histogram of further exploration at site II is shown in Figure 6. From Figure 6 we can see that the most populated cluster with 51 conformations has the second lower binding energy, so the lowest binding energy conformation of this cluster is selected and analyzed as the best binding mode. The best energy ranked results of the binding mode between TRES and BAS are shown in Figures 7 and 8 . From Figure 7, it can be seen that TRES is located within the binding pocket of subdomain IIIA (site II, the flufenamic acid binding pocket) of the protein and the two rings are not coplanar. In Figure 8, TRES molecule is surrounded by 27 amino acid residues within $6 \AA$ : 14 hydrophobic residues (Leu-387, Leu-407, Leu-453, Leu-430, Leu-457, Ile-388, Phe403, Phe-488, Gly-431, Gly-434, Val-433, Pro-486, Ala-449, 


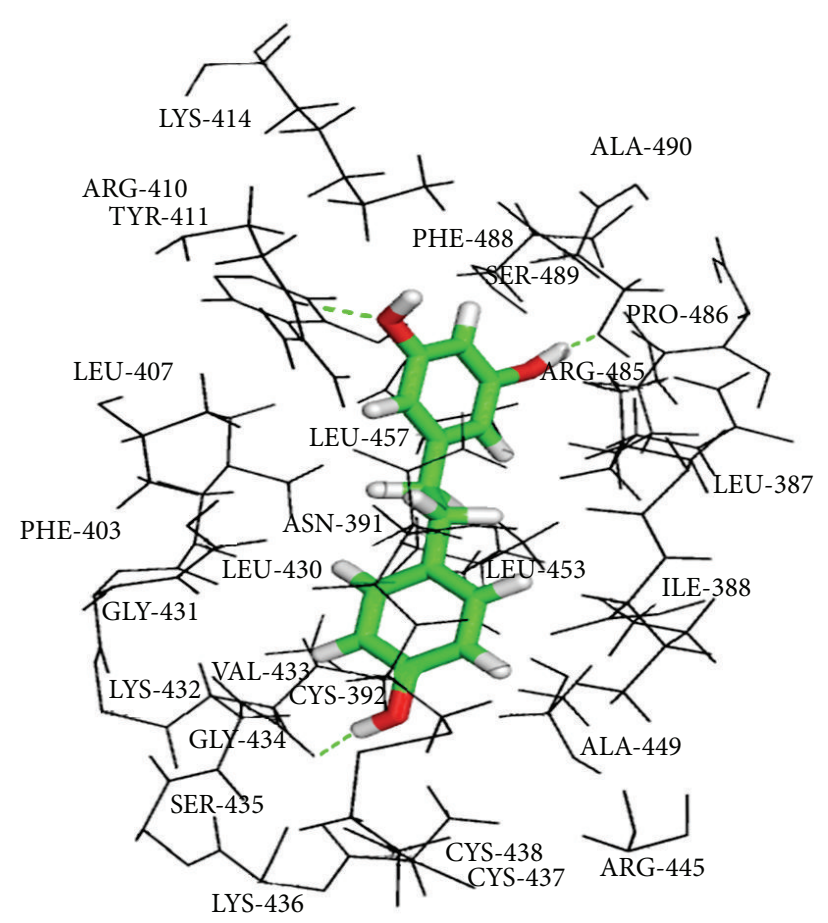

FIGURE 8: The amino acid residues around TRES within $6 \AA$. The residues of BAS are represented using line, TRES structure is represented using stick, and the hydrogen bonds are represented using green dashed line.

Ala-490), 7 hydrophilic residues (Asn-391, Cys-392, Cys-437, Cys-438, Tyr-411, Ser-435, Ser-489), and 6 ionic residues (Arg-410, Arg-445, Arg-485, Lys-414, Lys-432, Lys-436). It is important to note that the ring of TRES is adjacent to more hydrophobic residues, so hydrophobic forces may play major rule in the binding. Furthermore there are three hydrogen bonds between the hydroxyl groups of TRES and the amino acid residues of BSA; they are $3-\mathrm{OH}$ and Ser- 489 with a bond length of $2.0 \AA, 5-\mathrm{OH}$ and Arg-410 with a bond length of $2.2 \AA$, and $4^{\prime}-\mathrm{OH}$ and Val- 433 with a bond length of $2.0 \AA$. The formation of hydrogen bonds decreases the hydrophilicity and increases the hydrophobicity to stabilize the BSA-TRES system. In addition, electrostatic interactions may play a subordinate role in stabilizing the drug molecule due to the presence of the ionic and polar residues proximity to TRES. Therefore, it can be concluded that the interaction between TRES and BSA is dominated by hydrophobic forces, and there are also hydrogen bonds and electrostatic interactions, which is in good agreement with the results of binding mode study. The calculated binding Gibbs free energy change is $-29.95 \mathrm{~kJ} \mathrm{~mol}^{-1}(298 \mathrm{~K})$, which is close to the experimental value $\left(-28.10 \mathrm{~kJ} \mathrm{~mol}^{-1}, 297 \mathrm{~K}\right)$ to a certain degree.

\section{Conclusions}

Interaction mechanism of TRES with BSA has been investigated by fluorescence spectroscopic, UV-vis spectroscopic, FTIR spectroscopic methods and molecular modeling techniques. The results show that TRES interacts with BSA through a combination of static quenching. The interaction is a spontaneous process and is driven by enthalpy and entropy together. The main interaction forces are hydrophobic forces accompanied by hydrogen bonds and electrostatic interactions. The secondary structure of BSA is changed after bonding with TRES. TRES can bind to BSA at site II in subdomain IIIA according to displacement experiments and molecular modeling.

\section{Conflict of Interests}

The authors declare that they have no conflict of interests with each other, and they also have no conflict of interests with Huamei Bioengineering Company, Xian Sino-Herb Biotechnology Company, ChemSkcteh, or GAMESS software.

\section{Acknowledgments}

The authors gratefully acknowledge the financial support of this study by the National Natural Science Foundation of China (Grant no. 20975081) and the special fund for basic scientific research of central colleges, Chang'an University (CHD2012JC093).

\section{References}

[1] V. M. Rosenoer, M. Oratz, and M. A. Rothschild, Albumin: Structure, Function, and Uses, Pergamon Press, Oxford, UK, 1977.

[2] X. Guo, X. Han, J. Tong et al., "The investigation of the interaction between piracetam and bovine serum albumin by spectroscopic methods," Journal of Molecular Structure, vol. 966, no. 1-3, pp. 129-135, 2010.

[3] H. Larsericsdotter, S. Oscarsson, and J. Buijs, "Structure, stability, and orientation of BSA adsorbed to silica," Journal of Colloid and Interface Science, vol. 289, no. 1, pp. 26-35, 2005.

[4] M. Okawara, H. Katsuki, E. Kurimoto, H. Shibata, T. Kume, and A. Akaike, "Resveratrol protects dopaminergic neurons in midbrain slice culture from multiple insults," Biochemical Pharmacology, vol. 73, no. 4, pp. 550-560, 2007.

[5] S. Pervaiz and A. L. Holme, "Resveratrol: its biologic targets and functional activity," Antioxidants and Redox Signaling, vol. 11, no. 11, pp. 2851-2897, 2009.

[6] F. Z. Marques, M. A. Markus, and B. J. Morris, "Resveratrol: cellular actions of a potent natural chemical that confers a diversity of health benefits," The International Journal of Biochemistry \& Cell Biology, vol. 41, no. 11, pp. 2125-2128, 2009.

[7] L. Rivera, R. Morón, A. Zarzuelo, and M. Galisteo, "Longterm resveratrol administration reduces metabolic disturbances and lowers blood pressure in obese Zucker rats," Biochemical Pharmacology, vol. 77, no. 6, pp. 1053-1063, 2009.

[8] B. Burstein, A. Maguy, R. Clément et al., "Effects of resveratrol (trans-3,5, $4^{\prime}$-trihydroxystilbene) treatment on cardiac remodeling following myocardial infarction," Journal of Pharmacology and Experimental Therapeutics, vol. 323, no. 3, pp. 916-923, 2007.

[9] X. Li, H. Li, M. Liu, G. Li, L. Li, and D. Sun, "From guest to ligand-a study on the competing interactions of antitumor drug resveratrol with $\beta$-cyclodextrin and bovine serum albumin," Thermochimica Acta, vol. 521, no. 1-2, pp. 74-79, 2011. 
[10] X. L. Wei, J. B. Xiao, Y. F. Wang, and Y. L. Bai, "Which model based on fluorescence quenching is suitable to study the interaction between trans-resveratrol and BSA?" Spectrochimica Acta Part A, vol. 75, pp. 299-304, 2010.

[11] S. Cao, D. Wang, X. Tan, and J. Chen, "Interaction between trans-resveratrol and serum albumin in aqueous solution," Journal of Solution Chemistry, vol. 38, no. 9, pp. 1193-1202, 2009.

[12] J. R. Lakowicz, Principles of Fluorescence Spectroscopy, Plenum, New York, NY, USA, 2nd edition, 1999.

[13] T. G. Dewey, Biophysical and Biochemical Aspects of Fluorescence Spectroscopy, Plenum Press, New York, NY, USA, 1991.

[14] S. M. T. Shaikh, J. Seetharamappa, P. B. Kandagal, and D. H. Manjunatha, "In vitro study on the binding of anti-coagulant vitamin to bovine serum albumin and the influence of toxic ions and common ions on binding," International Journal of Biological Macromolecules, vol. 41, no. 1, pp. 81-86, 2007.

[15] B. H. M. Hussein, "Spectroscopic studies of 7, 8-dihydroxy-4methylcoumarin and its interaction with bovine serum albumin," Journal of Luminescence, vol. 131, no. 5, pp. 900-908, 2011.

[16] A. Belatik, S. Hotchandani, J. Bariyanga, and H. A. Tajmir-Riahi, "Binding sites of retinol and retinoic acid with serum albumins," European Journal of Medicinal Chemistry, vol. 48, pp. 114-123, 2012.

[17] P. Bourassa, I. Hasni, and H. A. Tajmir-Riahi, "Folic acid complexes with human and bovine serum albumins," Food Chemistry, vol. 129, no. 3, pp. 1148-1155, 2011.

[18] D. Leckband, "Measuring the forces that control protein interactions," Annual Review of Biophysics and Biomolecular Structure, vol. 29, pp. 1-26, 2000.

[19] P. D. Ross and S. Subramanian, "Thermodynamics of protein association reactions: forces contributing to stability." Biochemistry, vol. 20, no. 11, pp. 3096-3102, 1981.

[20] T. Kosa, T. Maruyama, and M. Otagiri, "Species differences of serum albumins: I. Drug binding sites," Pharmaceutical Research, vol. 14, no. 11, pp. 1607-1612, 1997.

[21] G. Sudlow, D. J. Birkett, and D. N. Wade, "Further characterization of specific drug binding sites on human serum albumin," Molecular Pharmacology, vol. 12, no. 6, pp. 1052-1061, 1976.

[22] I. Sjöholm, B. Ekman, A. Kober, I. L. Pahlman, B. Seiving, and T. Sjödin, "Binding of drugs to human serum albumin: XI. The specificity of three binding sites as studied with albumin immobilized in microparticles," Molecular Pharmacology, vol. 16, no. 3, pp. 767-777, 1979.

[23] Q. L. Zhang, Y. N. Ni, and S. Kokot, "Molecular spectroscopic studies on the interaction between Ractopamine and bovine serum albumin," Journal of Pharmaceutical and Biomedical Analysis, vol. 52, no. 2, pp. 280-288, 2010.

[24] J. Tian, J. Liu, X. Tian, Z. Hu, and X. Chen, "Study of the interaction of kaempferol with bovine serum albumin," Journal of Molecular Structure, vol. 691, no. 1-3, pp. 197-202, 2004.

[25] J. W. Brauner, C. R. Flach, and R. Mendelsohn, "A quantitative reconstruction of the amide I contour in the IR spectra of globular proteins: from structure to spectrum," Journal of the American Chemical Society, vol. 127, no. 1, pp. 100-109, 2005.

[26] P. Bourassa, S. Dubeau, G. M. Maharvi, A. H. Fauq, T. J. Thomas, and H. A. Tajmir-Riahi, "Locating the binding sites of anticancer tamoxifen and its metabolites 4-hydroxytamoxifen and endoxifen on bovine serum albumin," European Journal of Medicinal Chemistry, vol. 46, no. 9, pp. 4344-4353, 2011.
[27] D. Roy, S. Dutta, S. S. Maity et al., "Spectroscopic and docking studies of the binding of two stereoisomeric antioxidant catechins to serum albumins," Journal of Luminescence, vol. 132, no. 6, pp. 1364-1375, 2012.

[28] W. H. Gao, N. Li, Y. W. Chen et al., "Study of interaction between syringin and human serum albumin by multi-spectroscopic method and atomic force microscopy," Journal of Molecular Structure, vol. 983, no. 1-3, pp. 133-140, 2010.

[29] K. Paal, A. Shkarupin, and L. Beckford, "Paclitaxel binding to human serum albumin - automated docking studies," Bioorganic and Medicinal Chemistry, vol. 15, no. 3, pp. 1323-1329, 2007. 

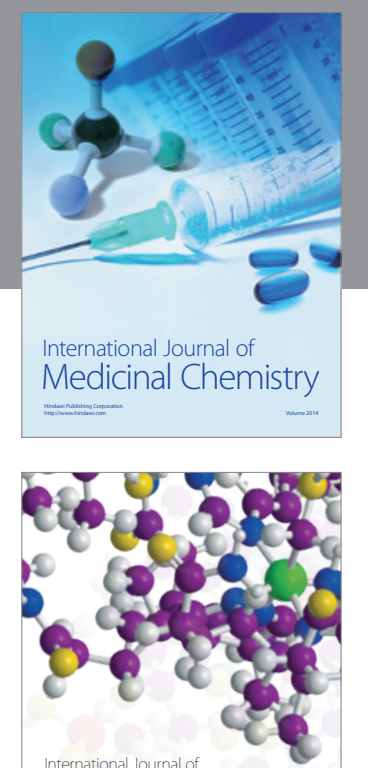

\section{Carbohydrate} Chemistry

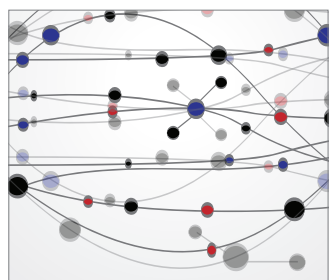

The Scientific World Journal
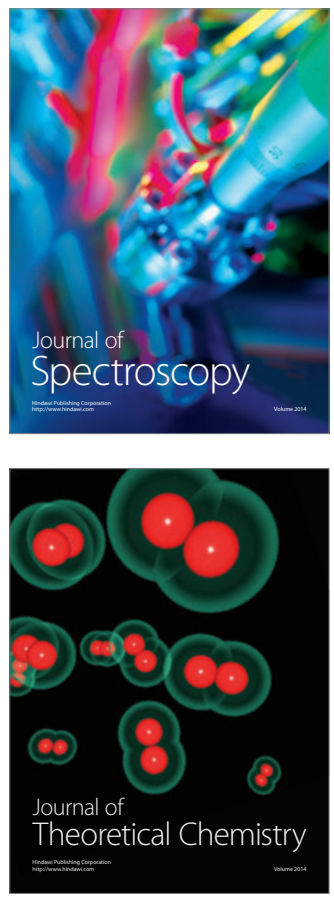
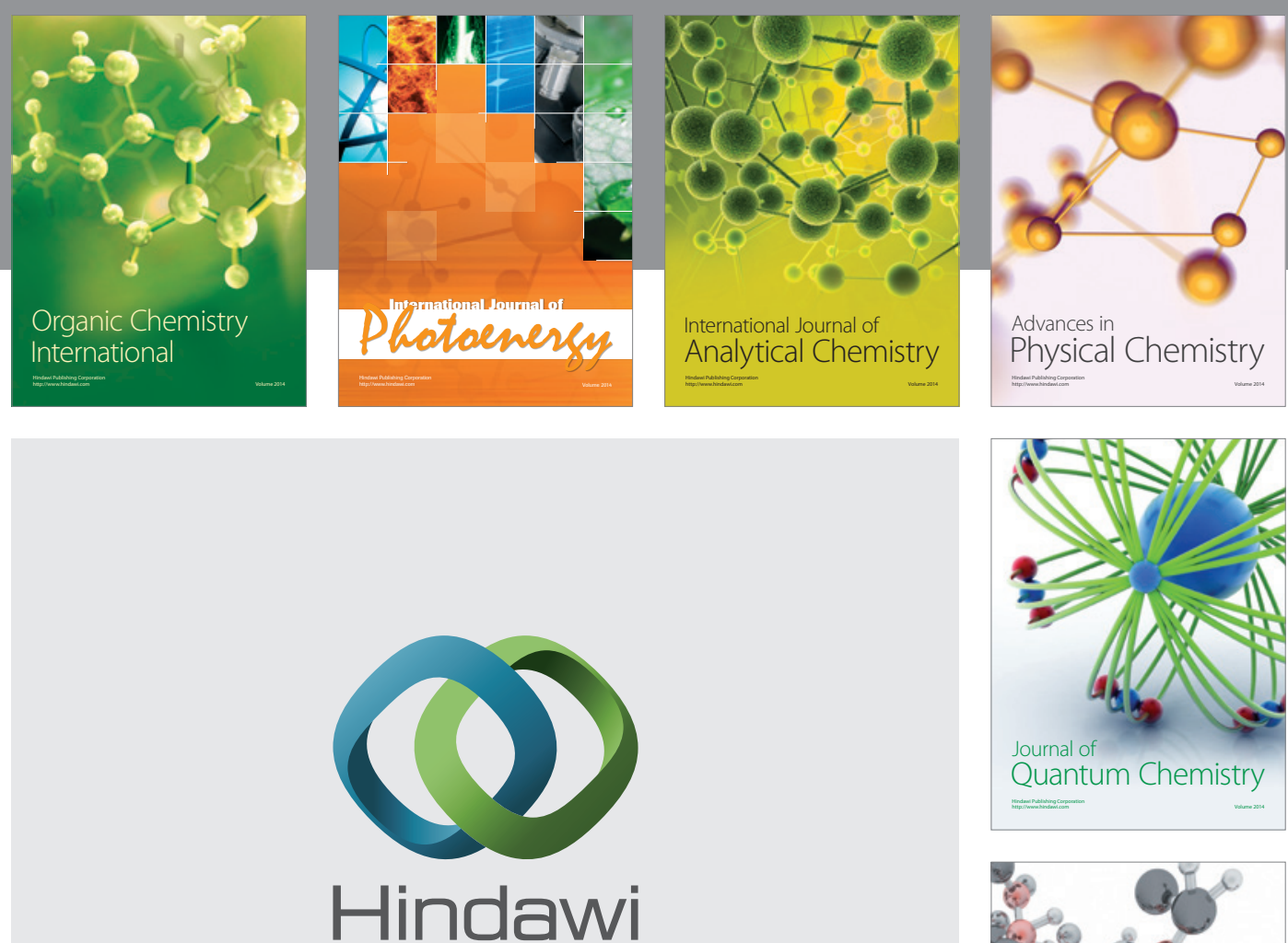

Submit your manuscripts at

http://www.hindawi.com

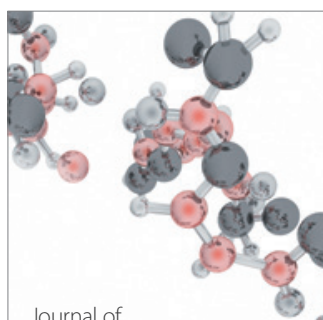

Analytical Methods

in Chemistry

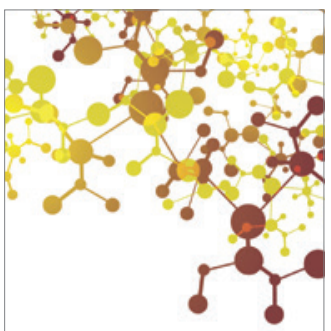

Journal of

Applied Chemistry

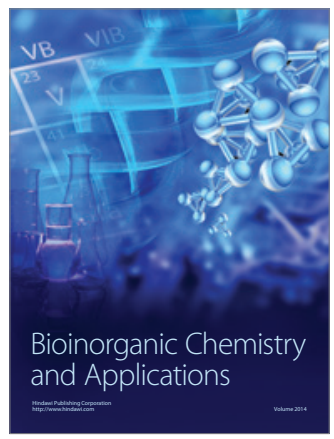

Inorganic Chemistry
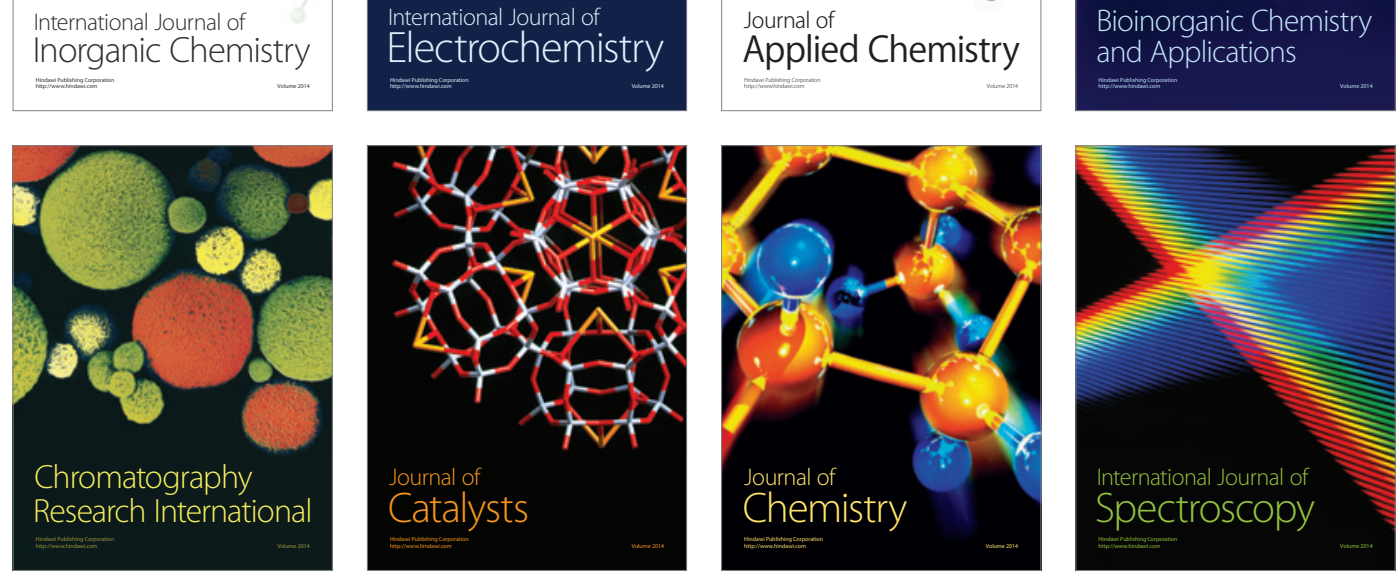